\title{
A Development of GPS SIS Anomalies Generation Software
}

\author{
Younghoon Han, Jaeyoung Ko, Mi Young Shin, Deuk Jae $\mathrm{Cho}^{\dagger}$ \\ Maritime Safety Research Division, Korea Institute of Ocean Science and Technology, Daejeon 305-343, Korea
}

\begin{abstract}
In this paper, GPS signal anomaly generation software is proposed which can be used for the analysis of GPS signal anomaly effect and the design, verification, and operation test of anomalous signal monitoring technique. For the implementation of anomalous signal generation technique, anomalous signals are generated using a commercial signal generation simulator, and their effects and characteristics are analyzed. An error model equation is proposed from the result of analysis, and the anomalous signal generation software is constructed based on this equation. The proposed anomalous signal generation software has high scalability so that users can easily utilize and apply, and is economical as the additional cost for purchasing equipment is not necessary. Also, it is capable of anomalous signal generation based on real-time signal by comparing with the commercial signal generation simulator.
\end{abstract}

Keywords: GPS signal anomaly, satellite clock anomaly, satellite orbit anomaly, anomalous signal generation, integrity monitor

\section{INTRODUCTION}

In recent years, the application fields and range of satellite navigation (e.g., ground transportation, maritime safety, and aviation safety) are rapidly increasing in Korea, and accordingly the demand and interest for the location information service and infrastructure based on more stable and reliable satellite navigation are also increasing. To secure the stability and reliability of the location information service based on satellite navigation, the monitoring of satellite signal anomalyis required, and thus the techniques for the satellite signal anomaly monitoring have continuously been studied (Shin 2013, Yoo 2011). Especially, as the signal anomaly in safetyrelated application fields could be directly linked to safety accidents, the satellite signal monitoring should be operated stably at all times. Therefore, test bed construction is needed for efficient technique design and various and continuous test and evaluation, and the anomalous signal generation

Received Mar 28, 2013 Revised Apr 19, 2013 Accepted Apr 22, 2013

†Corresponding Author

E-mail: djcho@kiost.ac

Tel: +82-42-866-3683 Fax: +82-42-866-3689 technique, which can generate signal anomalies of diverse scenarios, is particularly needed among the factors for the test bed construction. The example of the study based on the anomaly case that occurred in reality is the SVN 49 anomaly modeling (Fay 2011), and the example of anomalous signal generation technique based on hardware is the simulator developed by Spirent (Spirent 2008). The SVN 49 anomaly modeling only considered low sensitivity signals, and the simulator based on hardware can generate diverse anomalous signals but cannot identically simulate the characteristics of real-time signal, and additional cost is necessary for purchasing equipment.

Therefore, this paper proposes GPS signal anomaly generation software which can generate diverse signal anomalies and reflect the characteristics of real-time signal. The proposed anomalous signal generation software is easy to utilize and apply as the software has high scalability, and is economical as the additional cost for purchasing equipment is not necessary. In this paper, for the development of anomalous signal generation software, the anomalous signals are generated at diverse scenarios using a commercial signal generation simulator, and the characteristics and effects of anomalous signals are analyzed. Based on the result of analysis, the error from the 
anomalous signal is modeled in a generalized form, and anomalous signals are generated. This paper only considers the satellite clock anomaly and satellite orbit anomaly.

This paper is composed of 5 chapters. In Chapter 2, the causes and characteristics of satellite clock anomaly and satellite orbit anomaly are explained. In Chapter 3, the satellite clock and orbit anomalies which are generated using a commercial signal generation simulator are analyzed, and the error is modeled for the implementation of anomalous signal generation technique. In Chapter 4, the structure of anomalous signal generation software is explained, and the performance is verified. The summary and conclusion of the study results are included in Chapter 5.

\section{GPS SATELLITE SIGNAL ANOMALY}

\subsection{Satellite Clock Anomaly}

The GPS satellite clock typically uses cesium (Cs) and rubidium ( $\mathrm{Rb}$ ), and for cesium, vibration occurs at a frequency of $9,192,631,770 \mathrm{~Hz}$. The clock instability is occurred as an atomic clock is very sensitive to surrounding environment such as the Sun, the Earth's shadow, temperature change due to satellite motion, and gravity (Anja \& Lambert 2008). Therefore, the ground station, which manages and supervises the GPS satellite, uploads the information for satellite clock error correction to satellite, and the user estimates and compensates the satellite clock error using the bias $\left(\mathrm{a}_{\mathrm{f} 0}\right)$, drift $\left(\mathrm{a}_{\mathrm{f} 1}\right)$, and drift rate $\left(\mathrm{a}_{\mathrm{f} 2}\right)$ information included in navigation message and the Eq. (1) (Kaplan \& Hegarty 2006).

$$
\delta t_{d k}=a_{f 0}+a_{f 1}\left(t-t_{o c}\right)+a_{f 2}\left(t-t_{o c}\right)^{2}
$$

where $\delta t_{d k}$ is the satellite clock error, $t_{o c}$ is the clock data reference time, and $t$ is the current time epoch. However, if the ground station misestimates the information for the satellite clock error correction and the erroneous information is included in navigation message, the effect is reflected in the pseudorange between satellite and receiver. This case occurred at SVN 37 on Aug. 17, 2007, and it was observed that the pseudorange error increased up to $60 \mathrm{~m}$ (Gao 2009).

Another cause for the satellite clock anomaly is the GPS satellite atomic clock outage itself. If the atomic clock outage occurs, the GPS satellite could have a problem with the signal generation process including the navigation message and code generation, and induce the problems such as the absence of signal acquisition and the increase

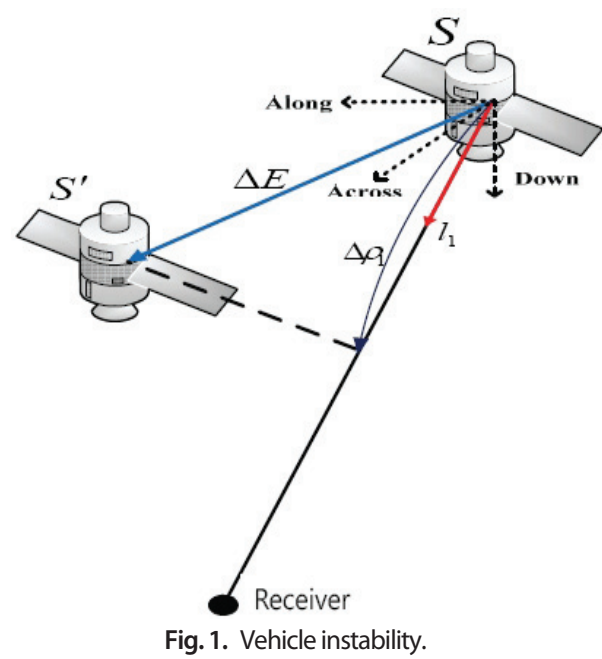

of pseudorange error due to the change in broadcasting frequency. There was a case that the rubidium clock of SVN 23 on Jan. 1, 2004, malfunctioned where the satellite signal was not acquired or an error of maximum $371 \mathrm{MHz}(70.6 \mathrm{~m} / \mathrm{s}$ pseudorange rate error) was observed at L1 frequency (Kim 2004).

\subsection{Satellite Orbit Anomaly}

The GPS satellite orbit can be estimated from 16 variables included in navigation message sub-frame 2 and 3 (Tsui 2000, IS-GPS-200 2001). Assuming that no abnormal effect occurred while the satellite signal is arriving at the user receiver, the circumstances that the user receiver misestimates a satellite orbit can be divided into two categories. First, it is when the ground station carried out the mismodeling for the satellite orbit. If the ground station uploads erroneous satellite ephemeris variables to satellite, the user receiver estimates a satellite orbit which differs from the true satellite orbit, and the effect is reflected in the pseudorange. This case actually occurred at SVN 40 on Feb. 8, 2007, and it was observed that the receiver showed similar operation to the Selective Availability (SA) (Gao2009). Second, it is when the satellite orbits erroneously. The satellite could orbit differently from what is expected due to the satellite deterioration and the abrupt change in space environment, and as shown in Fig. 1, a pseudorange change of $\Delta \rho$ occurs when there is a change of $\Delta \mathrm{E}$. The similar case actually occurred at SVN 56 , and the receiver showed similar phenomenon to the case that the ground station carried out the mismodeling for the satellite orbit (Jakab 2001). 


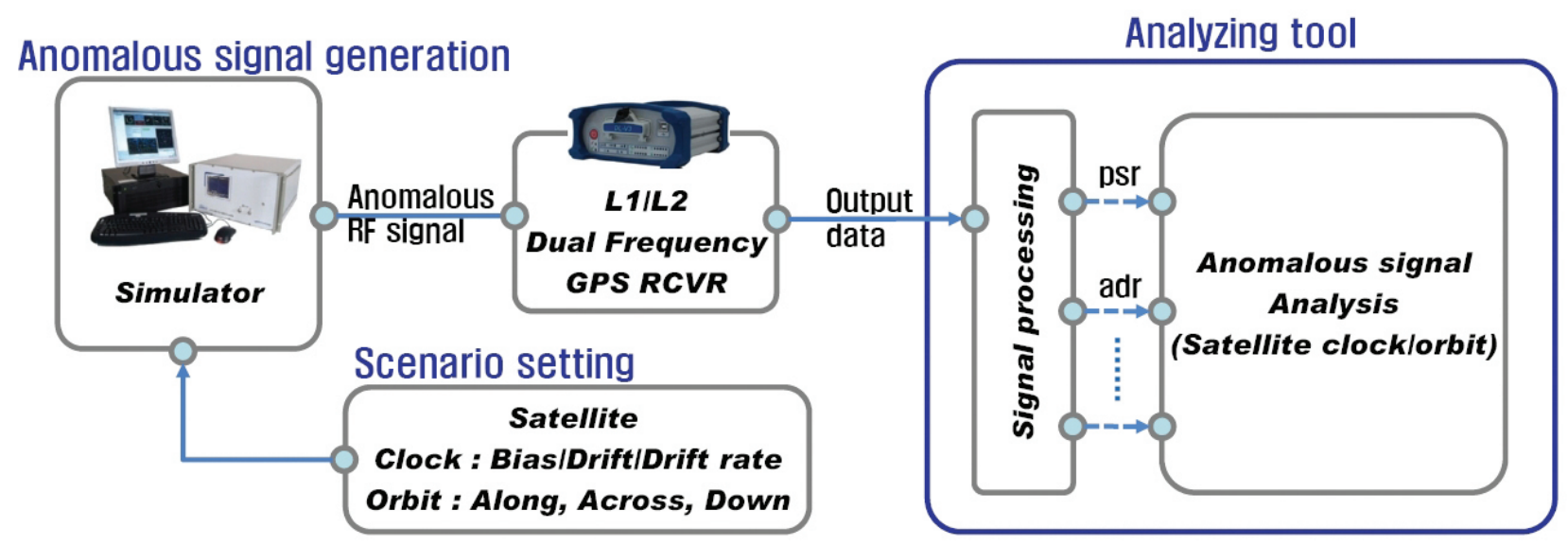

Fig. 2. Test environment of GPS SIS anomalies using simulator.

Table 1. Performance evaluation in dynamic tests.

\begin{tabular}{|c|c|c|c|}
\hline \multirow{8}{*}{$\begin{array}{l}\text { Satellite } \\
\text { clock } \\
\text { anomaly }\end{array}$} & \multirow{4}{*}{$\operatorname{Bias}\left(\mathrm{a}_{\mathrm{f} 0}\right)$} & \multicolumn{2}{|c|}{ 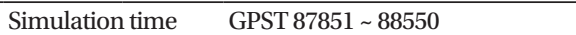 } \\
\hline & & \multicolumn{2}{|c|}{ Anomaly input time GPST 88201} \\
\hline & & \multicolumn{2}{|l|}{ Anomaly PRN } \\
\hline & & Error & $9 \times 10^{-9}[\mathrm{~s}](\cong 2.698132[\mathrm{~m}])$ \\
\hline & & Simulation time & GPST $173231 \sim 173930$ \\
\hline & \multirow{3}{*}{$\begin{array}{l}\text { Drift }\left(\mathrm{a}_{\mathrm{f} 1}\right) / \\
\text { Drift rate } \\
\left(\mathrm{a}_{\mathrm{f} 2}\right)\end{array}$} & \multicolumn{2}{|c|}{ Anomaly input time GPST 173581} \\
\hline & & Anomaly PRN & PRN 3 \\
\hline & & Error & $\begin{array}{l}1 \times 10^{-11}[\mathrm{~s} / \mathrm{s}](\cong 0.002997[\mathrm{~m} / \mathrm{s}]) \\
1 \times 10^{-13}\left[\mathrm{~s} / \mathrm{s}^{2}\right]\left(\cong 0.000029\left[\mathrm{~m} / \mathrm{s}^{2}\right]\right)\end{array}$ \\
\hline
\end{tabular}

Table 2. Satellite orbit anomaly scenario.

\begin{tabular}{lll}
\hline \multirow{2}{*}{$\begin{array}{l}\text { Satellite } \\
\text { orbit }\end{array}$} & Simulation time & GPST 87851 88550 \\
anomaly & Anomaly input time & GPST 88201 \\
& Error & PRN 3 \\
& & Along $(10[\mathrm{~m}])$, Across (10 [m]),Down (5 [m]) \\
\hline
\end{tabular}

\section{ANALYSIS OF GPS SIGNAL ANOMALY EFFECT AND MODELING OF ERROR}

\subsection{Test Environment}

The test environment for the analysis of GPS signal anomaly effect is shown in Fig. 2. The Spirent GSS8000 is used as the signal generation simulator, and the NovAtel DL-V3 is used as the receiver. The signal is generated using the signal generation simulator by setting the satellite clock anomaly and satellite orbit anomaly scenarios as shown in Tables 1 and 2, respectively. To examine the effects of satellite clock and orbit anomalies, the anomalies are permitted after the arbitrary time passes from the start time of signal generation. The anomalous signal generated from the simulator is collected using the receiver and goes through the signal processing procedure, and the receiver output variables such as pseudorange, Accumulated
Doppler Range (ADR), and satellite position are obtained from the receiver log file. This paper analyzes the effect of anomalous signal using the receiver output variables so that the users, who cannot control the parameter of receiver, are able to utilize, and the mean and variance of receiver output variables before and after the anomaly permission are compared. The error mentioned in this paper indicates the error which arises additionally from the satellite clock or orbit anomalies, and the cycle slip of carrier phase measurement is not considered.

\subsection{Satellite Clock Anomaly}

Fig. 3 and Table 3 show the analysis result of satellite clock anomaly effect at the scenario environment of Table 1. Fig. 3 shows the pseudorange and ADR errors of PRN3 due to the satellite clock anomaly, and the bias, drift, and drift rate type errors are observed after the anomaly permission. Table 3 summarizes the mean, variance, and satellite clock error for the intervals before and after the anomaly permission with regard to Fig. 3.

After the satellite clock anomaly permission, the means

Table 3. Performance evaluation in dynamic tests.

\begin{tabular}{|c|c|c|c|c|c|}
\hline & \multicolumn{3}{|c|}{ Mean } & \multicolumn{2}{|c|}{ Variance } \\
\hline & \multicolumn{2}{|c|}{ psr } & adr & psr & adr \\
\hline & before & after & before after & before after & before after \\
\hline$a_{f 0}$ & 0.008998 & -2.66838 & $0.008524 \quad 2.71345$ & $0.032518 \quad 0.031168$ & 0.0311680 .030958 \\
\hline error & 2.6773 & $78[\mathrm{~m}]$ & $2.704926[\mathrm{~m}]$ & $-0.000353[\mathrm{~m}]$ & $0.00021[\mathrm{~m}]$ \\
\hline$a_{f 1}$ & -0.00109 & -0.00559 & $0.001341 \quad 0.00533$ & $0.003510 \quad 0.003231$ & 0.0034730 .003215 \\
\hline error & 0.0035 & {$[\mathrm{~m} / \mathrm{s}]$} & $0.002989[\mathrm{~m} / \mathrm{s}]$ & $0.000279[\mathrm{~m}]$ & $0.000258[\mathrm{~m}]$ \\
\hline$a_{\mathrm{f} 2}$ & 0.00001 & -0.000013 & 0.0000140 .000036 & 0.0015920 .002038 & 0.0015530 .001961 \\
\hline error & 0.00002 & $3\left[\mathrm{~m} / \mathrm{s}^{2}\right]$ & $0.000022\left[\mathrm{~m} / \mathrm{s}^{2}\right]$ & $0.000446[\mathrm{~m}]$ & $0.000408[\mathrm{~m}]$ \\
\hline
\end{tabular}



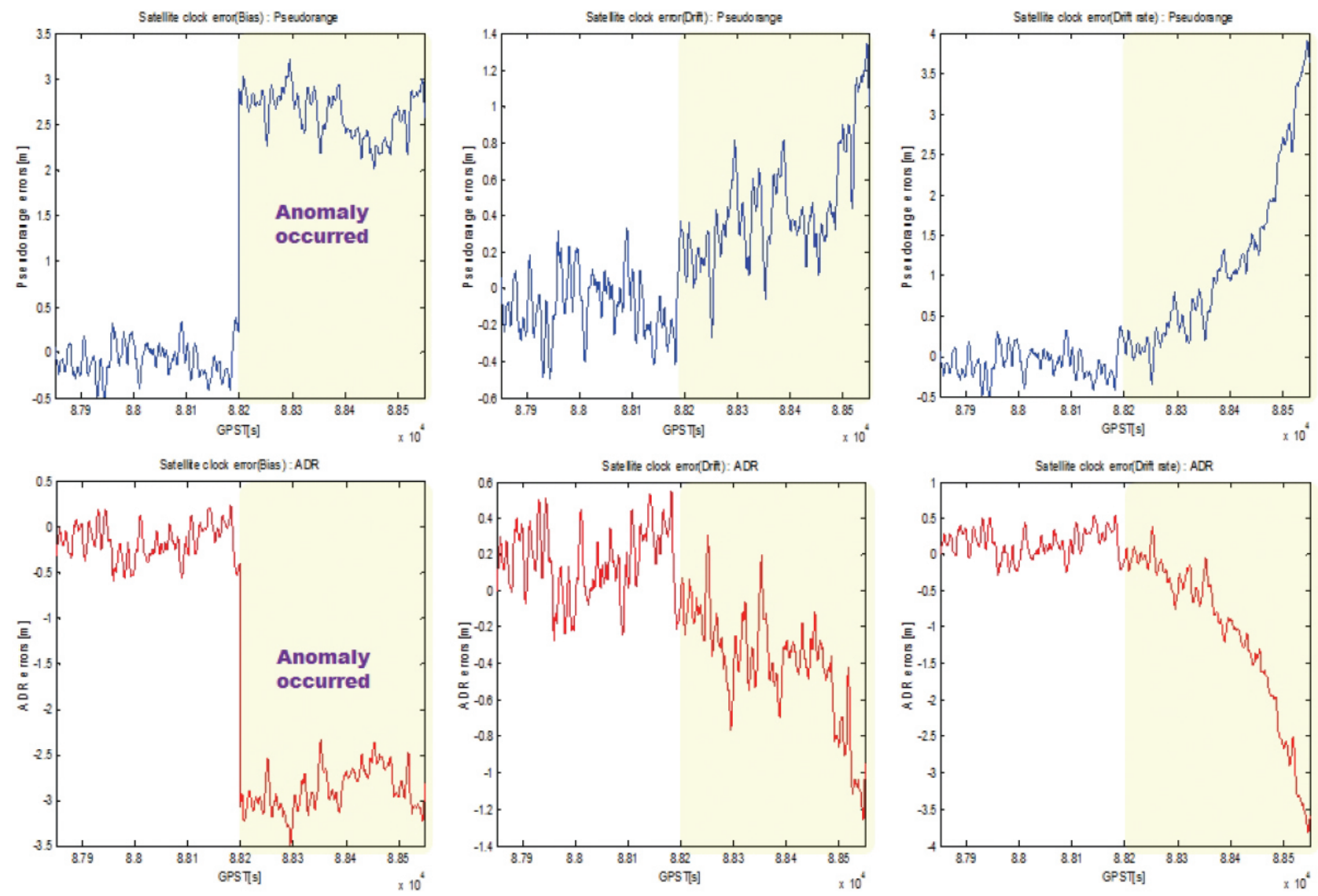

Fig. 3. Pseudorange errors(top) and ADR errors(bottom) of satellite clock anomalies.

for the pseudorange and ADR errors of PRN3 increase depending on the anomaly type, and the variances only showed a change of 0.00021 to $0.000446 \mathrm{~m}$. Therefore, the error model equation for the anomalous signal generation only reflects the characteristics of the mean. As for the difference between the permitted anomaly size using simulator and the clock error analyzed through the test, the pseudorange is $0.02 \mathrm{~m}$ and $\mathrm{ADR}$ is $0.006 \mathrm{~m}$ for the bias type, the pseudorange is $0.0005 \mathrm{~m} / \mathrm{s}$ and ADR is $0.000008 \mathrm{~m} / \mathrm{s}$ for the drift type, and the pseudorange is $0.000006 \mathrm{~m} / \mathrm{s}^{2}$ and ADR is $0.000007 \mathrm{~m} / \mathrm{s}^{2}$ for the drift rate type. Considering the receiver measurement noise, it is confirms that the pseudorange and ADR errors are observed as much as the permitted anomaly sizes. The satellite clock error model obtained from the result of effect analysis is shown in Eq. (2). Here, $t_{\text {in }}$ is the start time of anomaly permission and $t$ is the current time epoch. $\mathrm{a}_{\mathrm{f} 0}, \mathrm{a}_{\mathrm{f} 1}$, and $\mathrm{a}_{\mathrm{f} 2}$ are the user setting variables for the satellite clock signal anomaly generation.

$$
\operatorname{satclk}_{\mathrm{err}}[\mathrm{m}]=\mathrm{a}_{\mathrm{f} 0}+\mathrm{a}_{\mathrm{f} 1}\left(\mathrm{t}-\mathrm{t}_{\mathrm{in}}\right)+\mathrm{a}_{\mathrm{f} 2}\left(\mathrm{t}-\mathrm{t}_{\mathrm{in}}\right)^{2}
$$

\subsection{Satellite Orbit Anomaly}

Fig. 4 and Table 4 show the analysis result of satellite orbit anomaly effect at the scenario environment of Table 2. Fig. 4 shows the satellite position, pseudorange, and ADR errors of PRN3 due to the satellite orbit anomaly. The X, Y, and Z axes position errors of satellite are observed after the anomaly permission, and the error of each axis varies depending on satellite track. The accompanying effect is also observed in the pseudorange and ADR errors, and the detailed effect is shown in Table 4 . Table 4 summarizes the mean, variance, and satellite orbit error for the intervals before and after the anomaly permission with regard to Fig. 4 .

After the satellite orbit anomaly permission, the observed pseudorange and ADR errors of PRN3 have sizes of 4.05 $\mathrm{m}$ and $4.14 \mathrm{~m}$, and change to about $0.01 \mathrm{~m} / \mathrm{s}$ depending on satellite track. Therefore, it is confirms that for the satellite orbit anomaly, the orbit error from the erroneous orbit information occurs in the form of step function right after the anomaly occurrence and the orbit error varies depending on satellite track. This characteristic of orbit error 


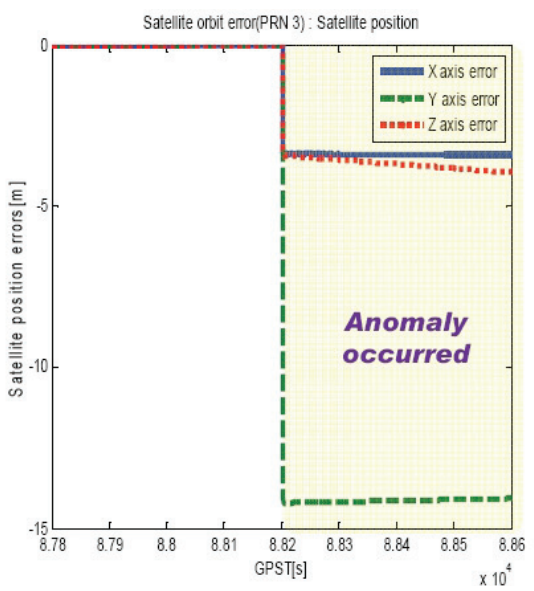

(a)

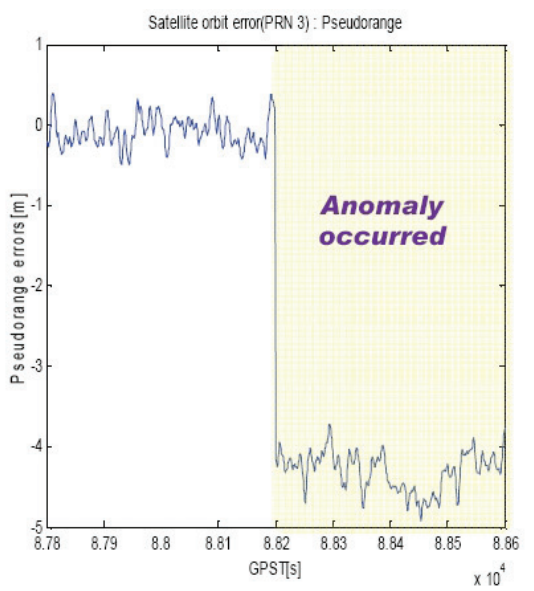

(b)

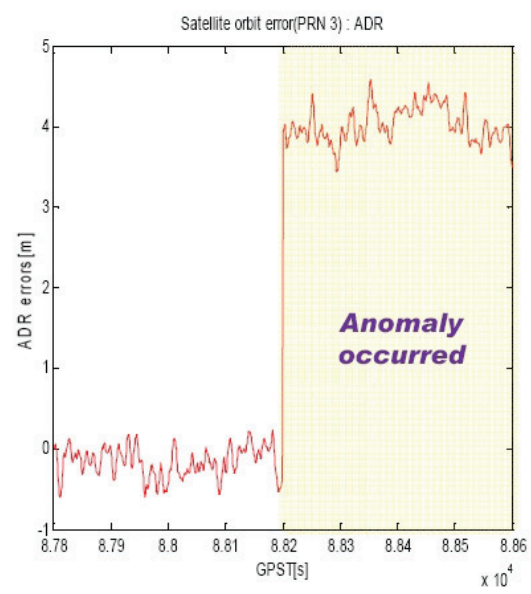

(c)

Fig. 4. Satellite position error(a), Pseudorange error(b) and ADR error(c) of satellite orbit anomaly.

Table 4. Mean and variance for pseudorange and ADR of PRN 3 (Satellite orbit anomaly).

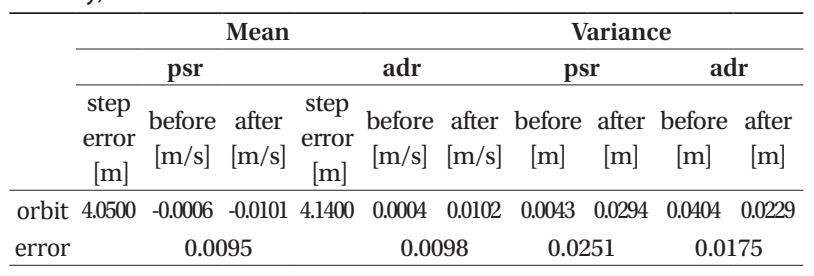

can be modeled as a first order function, and the satellite orbit error model obtained from the result of effect analysis is shown in Eq. (3).

orbit $_{e r r}[m]=\sqrt{\left(x_{s a t}(t)-x_{r c v}(t)\right)^{2}+\left(y_{s a t}(t)-y_{r c v}(t)\right)^{2}+\left(z_{s a t}(t)-z_{r c v}(t)\right)^{2}}$ $-\sqrt{\left(x_{s a t}(t)+x_{e r r}(t)-x_{r c v}(t)\right)^{2}+\left(y_{s a t}(t)+y_{\text {err }}(t)-y_{r c v}(t)\right)^{2}+\left(z_{s a t}(t)+z_{e r r}(t)-z_{r c v}(t)\right)^{2}}$

where $\mathrm{x}_{\text {sat }}(\mathrm{t}), \mathrm{y}_{\text {sat }}(\mathrm{t})$, and $\mathrm{z}_{\text {sat }}(\mathrm{t})$ represent the satellite position at current time epoch, $t . x_{r c v}(t), y_{r c v}(t)$, and $z_{r c v}(t)$ represent the receiver position. $x_{\text {err }}(t), y_{\text {err }}(t)$, and $z_{\text {err }}(t)$ are the satellite position errors relative to $\mathrm{X}, \mathrm{Y}$, and $\mathrm{Z}$ axes, which are shown in Eq. (4). $a_{x^{\prime}}, b_{x^{\prime}}, a_{y^{\prime}} b_{y^{\prime}} a_{z^{\prime}}$ and $b_{z}$ are the user setting variables for the satellite orbit signal anomaly generation. For the result of effect analysis in this study, the $a_{x^{\prime}} b_{x^{\prime}} a_{y}, b_{y^{\prime}} a_{z^{\prime}}$ and $\mathrm{b}_{\mathrm{z}}$ are -0.0001, -3.382, 0.0004, -14.206, -0.0013, and -3.437, respectively.

$$
\begin{aligned}
& x_{\text {err }}(t)=a_{x} t+b_{x} \\
& y_{\text {err }}(t)=a_{y} t+b_{y} \\
& z_{\text {err }}(t)=a_{z} t+b_{z}
\end{aligned}
$$

\section{ANOMALOUS SIGNAL GENERATION SOFTWARE}

\subsection{Structure of Anomalous Signal Generation Software}

Using the satellite clock and orbit errors analyzed and modeled in Chapter 3, the anomalous signal generation software which can permit an anomaly to a real-time signal was constructed, and its structure is shown in Fig. 5. In the input section, the satellite signal information collected using a commercial receiver is inputted, and from the input information, the pseudorange, ADR, and satellite position values which are necessary for the anomalous signal generation are classified. In the anomaly scenario setting section, the anomaly type and error size that are to be permitted are set. The anomaly type includes the satellite clock anomaly and satellite orbit anomaly. When the satellite clock anomaly is selected, the sizes of bias, drift, and drift rate errors included in Eq. (2) can be set, and when the satellite orbit anomaly is selected, the sizes of satellite position errors relative to $\mathrm{X}, \mathrm{Y}$, and $\mathrm{Z}$ axes $\left(\mathrm{a}_{\mathrm{x}}, \mathrm{b}_{\mathrm{x}^{\prime}}, \mathrm{a}_{\mathrm{y}^{\prime}} \mathrm{b}_{\mathrm{y}^{\prime}}, \mathrm{a}_{\mathrm{z}^{\prime}}\right.$, and $\mathrm{b}_{\mathrm{z}}$ ) included in Eq. (3) can be set. Also, it is possible to set the permission time and generation cycle of anomalous signal. In the error generation section, the errors for the anomaly scenario selected by the user are generated, and these errors are reflected in the pseudorange and ADR. In the output section, the pseudorange and ADR, in which the errors are reflected, are restored in the form of the raw signal collected at the receiver, and forwarded to the user.

\subsection{Performance Verification}

Fig. 6 shows the test environment for the performance 


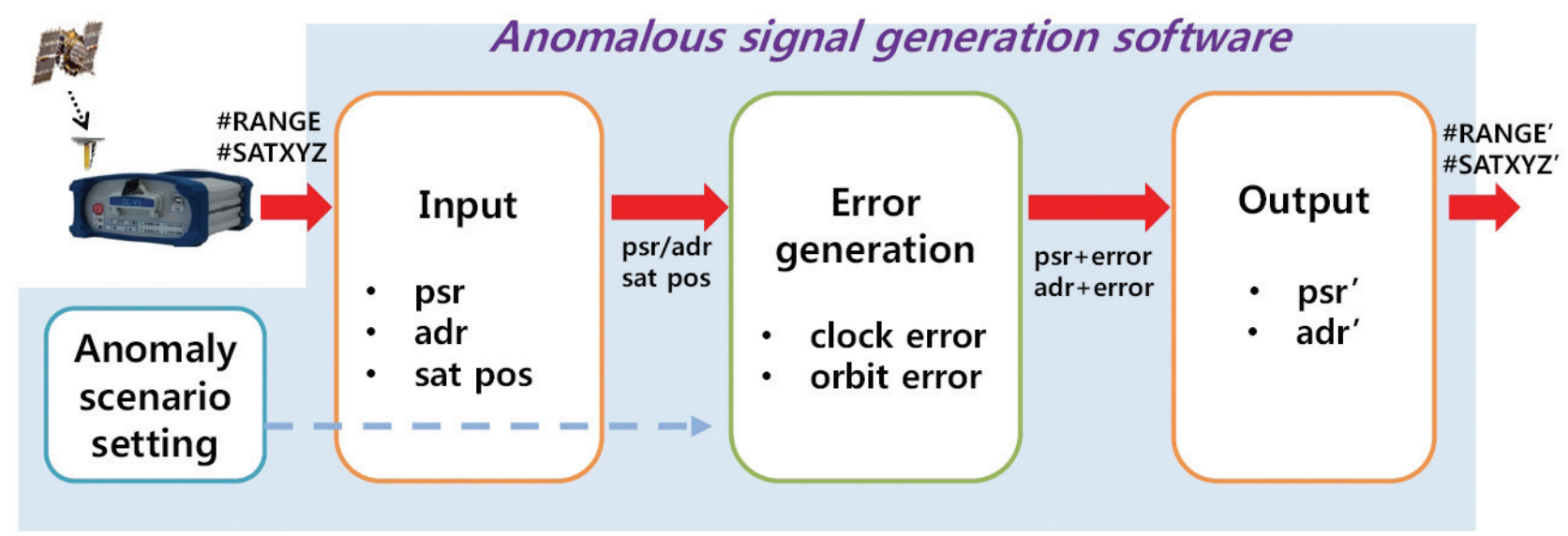

Fig. 5. The structure of anomalous signal generation software.

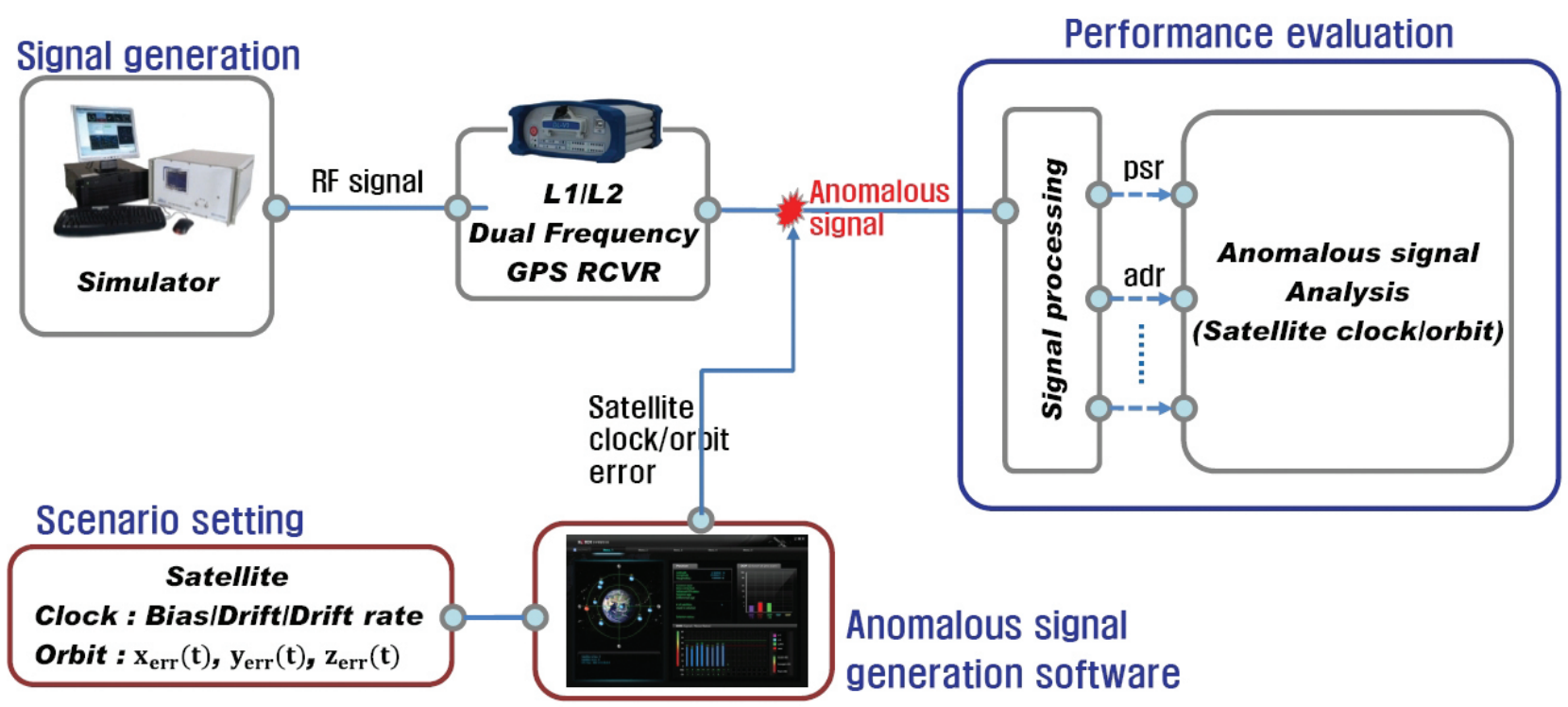

Fig. 6. Test environment for performance verification.

verification of anomalous signal generation software. In the same scenario environment as the effect analysis test in Chapter 3, the normal signal, to which an anomaly is not permitted, is generated using the commercial signal generation simulator, and the satellite clock and orbit anomalies for the scenarios of Tables 1 and 2 are permitted using the anomalous signal generation software. Then, by collecting the signal with the use of receiver, the pseudorange and ADR errors due to the satellite clock and orbit anomalies are analyzed and compared with the result of Chapter 3.

Table 5 summarizes the analysis result for the pseudorange and ADR errors of satellite clock signal anomaly generated using the anomalous signal generation software. In the analysis result of Chapter 3, considering the receiver

Table 5. Mean and variance for pseudorange and ADR of PRN 3 (Satellite clock anomaly generation by using proposed software).

\begin{tabular}{|c|c|c|c|c|}
\hline & \multicolumn{2}{|c|}{ Mean } & \multicolumn{2}{|c|}{ Variance } \\
\hline & psr & adr & psr & adr \\
\hline & before after & before after & before after & before after \\
\hline$a_{50}$ & -0.0782692 .628744 & $-0.166909-2.898395$ & 0.0558150 .058826 & 0.0569290 .048223 \\
\hline error & $2.707013[\mathrm{~m}]$ & $2.731486[\mathrm{~m}]$ & $0.003011[\mathrm{~m}]$ & $0.008706[\mathrm{~m}]$ \\
\hline$a_{f 1}$ & $0.000381 \quad 0.002810$ & $-0.000020 \quad-0.002764$ & $0.003032 \quad 0.002680$ & $0.002608 \quad 0.002486$ \\
\hline error & $0.002429[\mathrm{~m} / \mathrm{s}]$ & $0.002744[\mathrm{~m} / \mathrm{s}]$ & $0.000352[\mathrm{~m}]$ & $0.000122[\mathrm{~m}]$ \\
\hline$a_{f 2}$ & 0.0000110 .000045 & $-0.000012 \quad-0.000024$ & $0.001363 \quad 0.001212$ & $0.001127 \quad 0.000950$ \\
\hline error & $0.000034\left[\mathrm{~m} / \mathrm{s}^{2}\right]$ & $0.000036\left[\mathrm{~m} / \mathrm{s}^{2}\right]$ & $0.000151[\mathrm{~m}]$ & $0.000177[\mathrm{~m}]$ \\
\hline
\end{tabular}


Table 6. Mean and variance for pseudorange and ADR of PRN 3 (Satellite orbit anomaly generation by using proposed software).

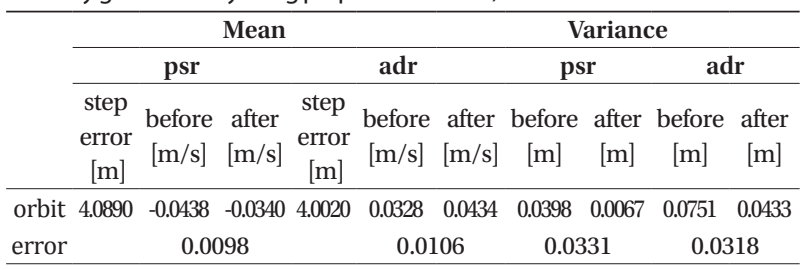

measurement noise, the pseudorange and ADR errors were observed as much as the anomalies permitted by the commercial signal generation simulator. A similar result was also confirmed in Table 5. As for the difference between the actually permitted anomaly size and the clock error analyzed through the test, the pseudorange is $0.008 \mathrm{~m}$ and ADR is $0.033 \mathrm{~m}$ for the bias type, the pseudorange is 0.000568 $\mathrm{m} / \mathrm{s}$ and ADR is $0.000253 \mathrm{~m} / \mathrm{s}$ for the drift type, and the pseudorange is $0.000005 \mathrm{~m} / \mathrm{s}^{2}$ and ADR is $0.000007 \mathrm{~m} / \mathrm{s}^{2}$ for the drift rate type.

Table 6 summarizes the analysis result for the pseudorange and ADR errors of satellite orbit signal anomaly. The result when the anomaly is permitted in the Along, Across, and Down axes relative to the satellite track and the result when the proposed satellite orbit error model equation is used show identical characteristics. Shortly after the satellite orbit anomaly permission, the pseudorange and ADR errors show differences of $0.039 \mathrm{~m}$ and $0.138 \mathrm{~m}$, respectively, and as for the satellite orbit errorvariation depending on satellite track, the pseudorange shows a difference of $0.003 \mathrm{~m} / \mathrm{s}$ and the ADR shows a difference of $0.008 \mathrm{~m} / \mathrm{s}$.

\section{SUMMARY AND CONCLUSION}

In this paper, GPS signal anomaly generation software was constructed. For the implementation of anomalous signal generation technique, the satellite clock and orbit signal anomalies were generated using the commercial signal generation simulator, and their effects and characteristics were analyzed. The result of analysis was presented as the error model equation for the anomalous signal generation in a generalized form. The performance of anomalous signal generation software was verified by generating anomalous signals in the same environment as the commercial signal generation simulator and analyzing the effects. The proposed anomalous signal generation software has high scalability so that users can easily utilize and apply, and has an advantage that the similar function to a commercial signal generation simulatoris available at low cost without adding hardware. Also, it can be used as a tool for the verification of anomalous signal monitoring technique based on real-time signal.

\section{ACKNOWLEDGMENTS}

This work was supported by the Ministry of Land, Infrastructure and Transport (06-A03).

\section{REFERENCES}

Anja, H. \& Lambert, W. 2008, Short-term Stability of GNSS Satellite Clocks and its Effects on Precise Point Positioning, Proceedings of the 21st International Technical Meeting of the Satellite Division of The Institute of Navigation, Savannah, GA, 16-19 Sep, pp. 1855-1863

Fay, G. 2011, Methodology for Modeling the SVN49 Anomaly for static scenarios, available from: http:// www.businesswire.com/news/home/20110118007047/ en/Spirent-Federal-SVN-49-Anomaly-Scenarios-Public

Gao, G. X. 2009, Methodology and Case Studies of Signalin-Space Error Calculation Top-down Meets Bottomup, Proceedings of the 22nd International Technical Meeting of The Satellite Division of the Institute of Navigation, Savannah, GA, 22-25 Sep, pp.2824-2831

IS-GPS-200, 2011, Navstar GPS Space Segment/Navigation User Interfaces, available from: http://www.navcen. uscg.gov/?pageName=gpsReferenceInfo

Jakab, A. J. 2001, Quality Monitoring Of GPS Signals, UCGE Reports, Number 20149

Kaplan, E. D. \& Hegarty, C. J. 2006, Understanding GPS Principles and Application, 2nd ed. (Lodon: Artech House)

Kim, J. W. 2004, Study on Fault analysis and detection of GPS PRN 23 Satellite, the 11th GNSS Workshop, Jeju

Shin, M. Y. 2013, Anomaly Detection Technique of Satellite on Network RTK, Journal of Navigation and Port Research, 37, 41-48

Spirent, 2008, GSS8000 Series - Datasheet: Capability, flexibility \& fidelity in GNSS simulation, MS3057

Tsui, J. B. 2000, Fundamentals of Global Positioning System Receivers: A Software Approach (New York: John Wiley \& Sons)

Yoo, Y. 2011, Detection algorithm of ionospheric delay anomaly based on multi-reference stations for ionospheric scintillation, Journal of Navigation and Port Research International Edition, 35, 701-706 


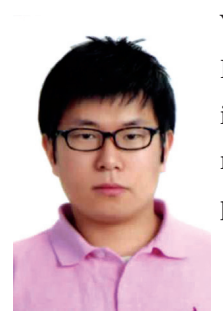

Younghoon Han received the Master's degree in Electronics from Chungnam National University in 2012. His research interests include on integrity monitoring, multipath mitigation techniques and precise positioning technology.

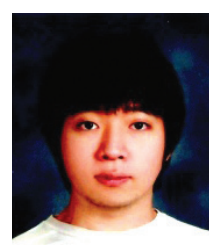

Jaeyoung Ko received the Master's degree in Electronics from Chungnam National University in 2012. His research interests include on antijamming techniques, integrity monitoring, and precise positioning technology.

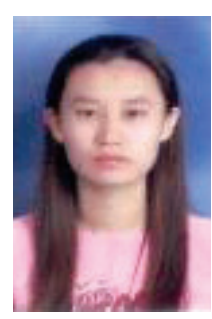

Mi Young Shin received the Doctor's degree in Electronics from Chungnam National University in 2011. Her research interests include antijamming and anti-spoofing techniques, eLoran, timing, integrity monitoring and precise positioning technology.

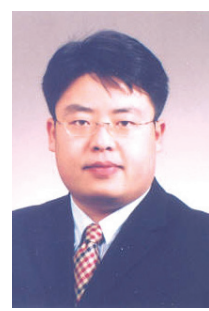

Deuk Jae Cho received the Doctor's degree in Electronics from Chung-nam National University in 2005. His research interests include software defined radio GNSS receiver, integrity monitoring, and precise point positioning technology. 